\title{
The Effectiveness of Relaxation Therapy in the Reduction of Anxiety Related Symptoms (A Case Study)
}

\author{
Uzma Ali, Ph.D (Corresponding author) \\ Associate Professor \\ Institute of Clinical Psychology, University of Karachi, Pakistan \\ 118, Block 20, Abul Asar Hafeez Jalindhri Road \\ Gulistan-e-Jauher-Karachi-Pakistan P.O Box 75290 \\ Tel : 92-300-921-0459_E-mail: druzmaali@yahoo.com
}

Shazia Hasan, Ph.D

Assistant Professor

Department of Management Sciences, COMSAT, Institute of Information technology

Riwid Road, Lahore, Pakistan

Tel : 92-321-920-1562Ｅ-mail: shazian36@yahoo.com (note 1)

\begin{abstract}
The aim of present paper is to highlight the significance of relaxation therapy for the reduction of anxiety related symptoms. This paper is based on a case of a girl who was suffering from fatigue and pain hampering her occupational and social life to certain level. Her problem remained unresolved through previous treatment. In psychological clinic psychological interventions of relaxation therapy were applied which included deep breath, instant vacation, ideal relaxation, visualization etc. IPAT Anxiety and IPAT Depression scale were administered before the start of therapy. Detailed history and psycho diagnostic report indicated that client could be diagnosed as having Anxiety Disorder NOS category according to the criteria of DSM-IV-TR. Subsequent to application of different techniques of relaxation therapy client also learned to do it herself at home. After twelve sessions both scales of anxiety and depression were administered again. Results showed drastic decrease in the level of anxiety and depression. Hence as reported by patient there was also reduction in muscle tension, pain, sleep disturbances and her occupational and social functioning were also restored.
\end{abstract}

Keywords: Relaxation therapy, Tense muscles, Anxiety, Depression occupational, Social functioning

\section{Introduction}

The term behavior is interpreted as covert responding of an individual for example, emotions, and verbalization; whereas when these can be clearly specified it is said to be overt responding. There are several behavioral techniques that have been employed since 1950s. Relaxation therapies, systematic desensitization, self monitoring, exposure, assertive training, modeling, self control procedures are its well known techniques (Rimm, 1979; Rodebaugh et al., 2004; Berstein, Borkovec, \&Hazlett, 2000).

There are individuals who suffer from anxiety disorders, such as generalized anxiety disorder, panic disorders with or with agoraphobia, and acute stress disorder; the symptoms of anxiety revolve around physiological symptoms like palpitation, sweating, muscular tension, pain, and headache. Besides physiological symptoms most of the clients with these disorders report pattern of thinking that shows thoughts and images of social or physical harm or both (Beck et al., 1974). There are some other clients who shared their concerns with diffuse pattern of worry and social failures (Chambless \&Gracely, 1989). These clients are best treated with cognitive behavioral therapies (Wright, 2006).

A variety of approaches have been used to modify cognitions (Ellis, 1962) and alter state of tension; progressive muscle relaxation (Jacobson, 1938; Wolpe 1973; Berstein et al., 2000) is the one of these approaches. A lot of empirical work (i.e Rimm, 1979;Clark et al., 2003; Conrad \&Roth, 2006; Goodwin \&Montgomery, 2006; Hoyer et al., 2009) showed that muscle tension is related to anxiety and that an individual will experience comfort and 
decreased amount of anxiety if tense muscles can be made loose and comfortable. Relaxation is best when used within the context of self -management by the clients. After the therapist has gone through the relaxation protocol two-three times, clients are encouraged to practice on their own, which serve as a mean of coping with stress.

Several researchers have worked on the effectiveness of relaxation therapy in the reduction of anxiety. Rokovec et al. (1987) found that cognitive behavior therapy was superior to nondirective plus relaxation therapy in student sample, while Borkovec and Mathews (1988) found that the effects of two treatments were similar at posttest and 12 months follow up with clinical sample. Clients with generalized anxiety disorder frequently show symptoms of depression Chambless and Gillis (1996, p.122) compared three studies of different researchers and found CBT to have greater impact on depression than behavior therapy (Butler et al., 1991); CBT was superior to nondirective therapy but not to applied relaxation (Borkovec \&Costello, 1993); further Borkovec et al.(1987) showed that there were no significantly superior CBT effects for depression among treatment completers. Further more Arakawa (1997) showed the efficacy of progressive muscle relaxation to reduce nausea, vomiting and anxiety induced by chemotherapy. Somewhat recently Hoyer et al. (2009) investigated the efficacy of worry exposure and applied relaxation therapeutic techniques for the treatment of generalized anxiety disorder, while comparing with control group both were found to be effective.

Considering above mentioned literature and applicability of relaxation therapy in psychologically disturbed individuals it is clear that relaxation therapy or cognitive behavior therapy plus relaxation are effective treatment methods in the reduction of depression and anxiety disorders. In our culture there is a lack of psychological sophistication in people, they think that symptoms are physiological in nature and only physician can treat it. If there is an absence of medical bases then people visit different types of healers who try to treat patients with conventional medicines. There are very few people who visit clinical psychologist for psycho therapy. However, with the advancement of education; now a day's people have started visiting clinical psychologist and for psychotherapy. In our culture mostly people don't express their feelings verbally; they tend to repress them, and when they couldn't express their wishes or desires in acceptable manner, they learn to express them in physiological symptoms. In the present study researchers want to understand the effectiveness of relaxation therapy for client who has anxiety symptoms that were accompanying depression and she was diagnosed with Anxiety Disorder Not other wise specified (DSM IV TR, APA, 2000).

Ms.X is a 20 years old girl who is a student of MS, in a well reputable institution at Karachi-Pakistan. She belongs to upper middle class family. Her father is working abroad (Middle East) and mother is a housewife. She is a first born and has one sister.

She came to Institute of Clinical Psychology, University of Karachi in private practice of the author with the presenting complaints of nervousness, irritability, crying spells, negative thinking, confused state of mind, uncertainty, guilt feeling, intra- aggression, disturbed sleep and appetite, indecisiveness, pain in legs and some times she becomes depressed. Detailed clinical interview revealed that her problem was started when she came to Karachi for her studies. She had been living abroad since her childhood but for her higher education she came to Pakistan since it was very expensive there. She was very close to her mother, but since the time she was living at Karachi (with her paternal grand parents) she felt anxious, lonely, had palpitation and become fearful.

She was also worried about her future especially about her male friend with whom she talks on phone. She had some traumatic experiences (sexual) in her childhood as she reported. She did not tell this to her mother. Her parents didn't have good relations since their marriage. She also felt that her father gave more appreciations to her younger sister while most of the time he criticized her. She was a good student in school. She had very good relations with her friends but somehow she felt fearful. Her sexual desires were sometimes disturbing for her, she wanted to control them but at times she did not succeed. These facts and perception were contributing to her extreme guilt, sadness, laziness and at times her absence from classes. She also took some medications by herself for her sleep problem.

The following tests were administered for her psychological evaluation, for identifying the problem area, estimation of prognosis and for treatment planning.

- Intake Card and Case History Sheet of Institute of Clinical Psychology, University of Karachi.

- Bender Gestalt Test (BG- Pascel, 1951); Human Figure Drawing Test (HFD-Koppitz, 1968; \&Gilbet, 1986);Thematic Apperception Test (TAT-Murray,1993) and the Rorschach Inkblot Test (ROR-Exner,2004). 
In conclusion it appeared that there were some anxieties and depressive feelings that were produced by external sources and due to her internal conflicts. She had intellectual potentials and some meaningful relations to deal with them and appeared less subject to distortion as she was able to perceive things in realistic and conventional way. However she also had extreme introspective tendencies and criticism towards self. Her feelings of inferiority and of being scared were due to her negative self image; which was developed after facing the traumatic experiences and criticism by others.

\section{Method}

\subsection{Participant}

Current research utilized the case study method $(\mathrm{n}=1)$. The characteristics, demographics and other related information are given in introduction.

\subsection{Measures}

\subsubsection{Intake Card and Case History Sheet:}

Intake Card and Case History Sheet of Clinical Psychology, University of Karachi is comprised of Identifying Information, family history, medical history, school and work history, friendship and other activities. It also focuses on information regarding sleep, orientation, behavior, and affect etc.

\subsubsection{IPAT Anxiety Scale: King, Scheier \& Cattell (1976)}

The total scores on anxiety scale contain 40 items. 20 items on the left hand test page are for unrealized, covert anxiety. 20 right hand test page items measures an overt, symptomatic, conscious anxiety. The scores are presented for covert (A) and overt (B) anxiety. This scale measures five components of personality, apprehension, tension, low self-Control, emotional instability and suspicion.

Scoring: for each item there are three options A, B, and C, B is always score as 1; where as A and C are score as 0 or 2 depending upon the question.

\subsubsection{IPAT Depression Scale: (Krug \& Laughlin, 1976)}

IPAT Depression scale, measuring depression, has 40 items 20 items on sheet A and 20 item on sheet B. the last two items on both of the sheet also evaluate anxiety symptoms. For taking depression score, clinician is required to minus the score of item no. 19, 20, 39 and 40. Now only depression score can be calculated this score is called "with correction factor". For present study depression scores were taken with correction factor.

Scoring: for each item there are three options A, B, and C, B is always score as 1; where as A and C are score as 0 or 2 depending upon the question.

\section{Therapeutic Intervention}

Initially the researcher took permission from the authorities of the Institution and discussed ethical consideration for this research. Then the informed consent was taken from the participant, and anonymity of identifying information was assured. Brief description of the research was also given. Therapy session was planned with the client. It was decided that twice a week sessions would be suitable. First of all therapist conducted a detail Interview, followed by a detailed psychological assessment and then the administration of IPAT Anxiety and IPAT depression Scales. In the initial sessions therapist facilitated the client to pent-up her emotions with the help of emotional catharsis, then the therapist noted the initial subjective evaluation of her symptoms, (like sleep disturbances, nervousness, feeling inferiority, inability to control sexual desires, examination anxiety and of being scared etc.) that may contribute in anxiety and depressive feelings. The client rated them between $0-100 \%$ intensity levels, along this cognitive restructuring was also done to change her negative thinking into positive one to decrease her depression. As she was also depressed, application of relaxation in the state of depression was not suitable. After 3-4 sessions depressive symptoms were decreased to some extent. For the reduction of her anxiety symptoms relaxation therapy was applied. Relaxation therapy was consisted of different techniques including deep breathe, simple muscle relaxation, walk, recreational activities, like watching favorite TV programs, talking on telephone with friend, doing household work and reading books. To reduce stress, time management was also done which was also helpful for sleep management and hence reduces laziness and fatigue. After the management of time and sleep, deep breathe and simple muscle relaxation were applied by the client herself at home.

The whole treatment interventions were consisted of 15 sessions; out of 15 sessions relaxation therapy was applied in 12 sessions. After the completion of 12 sessions IPAT Anxiety and IPAT Depression Scale were administered again to evaluate the symptoms of anxiety and depression. Subjective ratings of above listed 
problems were also done. To evaluate follow up sessions the therapist called the client and she reported that she is functioning pretty well and also attained A grade in exams, however due to her visit to abroad she could not manage to come in follow up sessions, so the overall treatment period was of 8 months.

\section{Results}

Therapeutic outcome of Relaxation therapy showed that relaxation techniques are very effective in the reduction of anxiety and depressive symptoms (Table I-III).

\section{Therapeutic Outcome}

Relaxation therapy is used with psychologically disturbed clients in clinical practice. This case study showed its effectiveness (see table I \& III) it is indicated that there was a reduction in anxiety related symptoms after applying the relaxation therapy. It included deep breathe, simple muscle relaxation, involving in recreational activities, going on vacations, managing time, and visualization. Client also verbalized the improvement during therapy sessions like "now I am attending university on time, and concentrating more on reading, things have settled now, I plan to sleep early so in morning I don't feel pain in my legs, feel less lethargic." She further reported that "now I am concentrating more on my studies and in my exam I did not forget I do most of the paper". The client's verbalization and pre-post difference in scores of depression, anxiety and subjective rating of anxiety related symptom showed the association between psychological problems and psychological intervention i.e. cognitive restructuring and relaxation therapies.

There was a marked decreased in depression (see Table II). It was further confirmed that (see table III) patient felt improvement in her negative thinking that was related to feeling of inferiority which was dealt with cognitive restructuring as according to Beck (Sharf, 2000) feelings of inferiority and worthlessness is a core belief that lead to depression.

After developing positive concept about self the connection between bodily tension and how to relax her was described. Here Relaxation techniques were very helpful in managing her sleep, nervousness, inability to control her desires. Other behavioral activities were a part of relaxation therapies that were applied by the client like she started involving herself in reading books, preparing daily meal, giving tuitions to children so that she become more busy in constructive activities and feel less anxious and depressed and her self image become positive as she said "I have done it that means if I work hard I can do it". This further showed that the outcome was clearly due to relaxation therapies that pushed her to constructive and relaxing activities. As Deffenbacher et al. (1988) suggested that preceding cognitive intervention with relaxation made them more acceptable and easier to implement.

Relaxation therapy is also useful in developing her perception of home environment as she said "now I feel less tension and also give time to mother, now relationships with father are also better, my father gave me time, I also communicated with him, I did not feel afraid." This change is also attributed to relaxation therapy because whenever she talked with her father she become tense, however after learning relaxation it was easier to communicate with her father as she reported.

One of her belief, feelings scared, that was based on catastrophizing cognitive distortion (Sharf, 2000) was also decreased, but it is still present to certain extent that is related to her fear of relapse as she said "I feel sometime fearful if I couldn't control my self, I am afraid if I loose my control and go back to previous state". To change this belief cognitive restructuring and relaxation were really helpful, however for this client was suggested to take further sessions.

Client's scores and subjective expression showed that relaxation therapy is very useful and effective in the reduction of anxiety symptoms and problem related to the areas that cause anxiety and depression. Our findings are also supported by the findings of Goodwin and Montgomery (2006); Conrad and Roth (2007) and Jacobson and Heather (2008).

\section{Conclusion and future directions}

It is concluded that relaxation replaces arousal, the client gains therapeutic outcome that involves a sense of control over disruptive emotional -physiological arousal, which helps her in restoring social occupational functioning. Relaxation therapies are found to be the key factor in the treatment of anxiety disorders. Present study demonstrates the effectiveness of psychological intervention in Pakistani (Asian) culture where people express their feelings in bodily symptoms. Considering the present finding it is strongly recommended that professionals dealing with mental disorders should consider the important of relaxation therapies in their treatment plan to rehabilitate clients' past functioning and to reintegrate their mental resources through different modalities. 
To generalize the findings further research should focus on the investigation of effectiveness of relaxation and cognitive behavior therapies with larger samples.

\section{Acknowledgement}

We are highly thankful to Dr. Riaz Ahmad, Associate Professor/Director Institute of Clinical Psychology, for giving permission to conduct this study and use the file record of the participant. We appreciate Prof. Dr. Seema Munaf, Institute of Clinical Psychology, and University of Karachi for her expert guidance in writing this paper.

\section{References}

American Psychiatric Association. (2000). Diagnostic and statistical manual of mental disorders, 4th ed., text revision. Washington, D.C.: Auther, American Psychiatric Association.

Arakawa,S. (1997). Relaxation to reduce nausea, vomiting, and anxiety induced by chemotherapy in Japanese patients. Cancer nursing, 20(5), 342-349.

Beck, A.T., Laude, R., \& Bohnert, M. (1974). Ideational components of anxiety neurosis. Achieves of General Psychiatry, 31, 319-325.

Berstein, D.A., Borkovec, T.D., \& Hazlett-Stevens, H. (2000). New directions in progressive relaxation training: A guidebook for helping professionals. Westport Conn: Praeger Publishers

Borkovec, T.D., \& Costello, E. (1993). Efficacy of applied relaxation and cognitive behavior therapy in in the treatment of generalized anxiety disorder. Journal of Counseling and Clinical Psychology, 61, 611-619.

Borkovec, T.D., \& Mathews, A.M. (1988).Treatment of nonphobic anxiety disorders: A comparison of nondirective, cognitive, and coping desensitization therapy. Journal of consulting and clinical Psychology, 56, 877-884.

Borkovec, T.D., Mathews, A.M., Chambers, A., Ebrahimi, S., Lytle, R., \& Nelson, R. (1987). The effects of relaxation training with cognitive or nondirective therapy and the role of relaxation-induced anxiety in the treatment of generalized anxiety. Journal of Counseling and Clinical Psychology, 55, 883-888.

Butler, G., Fennell, M., Robson, P., \& Gelder, M. (1991). Comparison of behavior therapy and cognitive behavior therapy in the treatment of generalized anxiety disorder. Journal of Counseling and Clinical Psychology, 59, 167-175.

Chambless, D.L., \& Gillis, M.M. (1996). Cognitive therapy of anxiety disorders. In K.S., Dobson. , \& Craig, K.D. (1996). Advances in Cognitive-Behavioral Therapy, (116-144), Sage publications: India.

Chambless, D.L., \& Gracely, E.J. (1989). Fear of fear and the anxiety disorders. Cognitive Therapy and Research, 13, 9-20.

Clark, D.M., Ehlers, A., McManus, F., Grey, N., Wild, J., \& Hackmann, A., et al., (2003). Cognitive therapy vs. exposure and applied relaxation in the treatment of social phobia: immediate outcome and mechanism of change. In S. Bogels, \& S. G. Hoffman (Eds).New developments in the treatment of social phobia: Mechanism of action and client characteristics.symposium conducted at the meeting of the association for advancement of behavior therapy, Boston.

Conrad, A., \& Roth, W. (2006). Muscle relaxation therapy for anxiety disorder: It works but how? Journal of Anxiety Disorder, 21(3),243-264.

Deffenbacher, J.L., Story, D.A., Brandon, A.D., Hogg, J.A., \& Hazaleus, S.L. (1988). Cognitive and Cognitive -relation treatments of anger. Cognitive Therapy and Research, 12, 167-184.

Ellis, A. (1962). Reason and emotion in psychotherapy. New York: Lyle Stuart.

Exner,Jr.J.E. (2004). A Rorschach workbook for the comprehensive system ( $5^{\text {th }}$ edi) Rorschach worshops-North Carolina.

Gilbet, J. (1986). Interpreting psychological research data; Vol. II Behavioral Attribute Antecedent. Litton Educational Publishing, In.

Goodwin, E.A., \& Montgomery, D.D. (2006). A Cognitive-Behavioral, Biofeedback-Assisted Relaxation Treatment for Panic Disorder With Agoraphobia. Clinical Case studies, 5(2), 112-125.

Hoyer, J., Beesdo, K., Gloster, A.T., Runge, J., Hofler, M., \& Becker, E.S. (2009). Worry Exposure versus Applied Relaxation in the treatment of generalized anxiety disorder. Psycother psychosom, 78, 106-115.

Jacobson, E. (1938). Progressive Relaxation. Chicago; University of Chicago Press. 
Jacobson, P.B., \& Heather, S.J. (2008). Psychosocial Interventions for Anxiety and Depression in Adult Cancer Patients: Achievement and Challenges.CA A cancer Journal of Clinicians, 58,314-230.

Koppitz,K.M. (1968). Psychological Evaluation of Children's Human Figure Drawing (HFD). Grune and Stratton, Inc,New York. U.S.A.

Krug, S. E., \& Laughlin, J. E. (1976). IPAT Depression Scale: Institute for personality and ability testing. Champaign,Illnois.

Krug., S. E., Scheier, I.H., \& Cattell,R.B. (1976). Hand book for the IPAT Anxiety Scale. Institute for Personality and ability testing, Inc. Champaign, Illinois, USA. pp.39-46.

Murry,A.M. (1943). Thematic Apperception test Manual (TAT). President and Fellows of Harvard College, press U.S.A.

Pascel,G.R., \& Shuttell,B.J. (1951). The bender -Gestalt Test: Quantification and validity for adult. Grune\&Statton,U.S.A.

Rimm, D.R. (1979). Behavior Therapy: Techniques and empirical findings (2 edi). Academic press Harcourt Brace Jovanovich, publishers, 1, 35.

Rodebaugh, T.L., Holaway, R.M., \& Heimberg, R.G. (2004).The treatment of Social anxiety disorder. Clinical Psychology Review, 24,883-908.

Rokovec, T.D., Mathews, A.M., Chambers, A., Ebrahimi, S., lytle,R., \& Nelson,R. (1987). The effects of relaxation training with cognitive or nondirective therapy and the role of relaxation-induced anxiety in the treatment of generalized anxiety disorder. Journal of Consulting and Clinical Psychology, 55, 883-888. In D.L, Chambless \& M.M.Gillis(1996).Cognitive Therapy of Anxiety Disorders.In K.S.Dobson.,\& K.D.Craig(Eds) (1996). Advances in Cognitive-Behavioral Therapy, (116-144), Sage publications, India.

Sharf. R. (2000). Theories of Psychotherapy \&Counseling: Concept and Cases( $2^{\text {nd }}$ Ed). Worsworth Thomson Learning, Belmont, USA. p.375-381.

Wolpe, J. (1973). The Practice of behavior therapy ( $2^{\text {nd }}$ ed). Oxford: Pergamon

Wright, J.H. (2006). Cognitive Behavior Therapy: Basic Principles and Recent Advances. Focus, 4:173-178.

\section{Notes}

Note 1. Coauthor Dr. Shazia Hasan, Assistant Professor was a faculty member at Institute of Clinical Psychology, University of Karachi during the time period in which this research was conducted.

Note 2. The Author presented this paper in 1st International Conference of Psychological Sciences and Application in March, 2010 Organized by United Arab Emirates University -Al Ain UAE.

Table 1. Pre and Post treatment raw score and std. score of IPAT Anxiety Scale

\begin{tabular}{l|l|l|l}
\hline & Pre treatment Score & Post treatment Score & pre-post difference \\
\hline$N=1$ & & & \\
Raw scores & 76 & 35 & 41 \\
Std. Scores & 10 & 77 & 03 \\
\hline
\end{tabular}

Note: Pre-post difference showed that after the application of relaxation therapy that there is a marked reduction in the scores of anxiety

Table 2. Pre and Post treatment raw score and sten score of IPAT Depression Scale

\begin{tabular}{l|l|l|l}
\hline & Pre treatment Score & Post treatment Score & pre-post difference \\
\hline$N=1$ & & & \\
Raw scores & 63 & 13 & 50 \\
Std. Scores & 10 & 04 & 06 \\
\hline
\end{tabular}

Note: Pre-post difference showed after the application of relaxation therapy that there is a marked reduction in the scores of depression 
Table 3. Client's subjective rating of her anxiety and depression related problems in pre-and post treatment

\begin{tabular}{l|l|l|l}
\hline Problem areas & pretreatment $\%$ & post treatment $\%$ & pre-post difference $\%$ \\
\hline 1. Nevousness & $80 \%$ & $30 \%$ & $50 \%$ \\
2. Sleep disturbances & $80 \%$ & $20 \%$ & $60 \%$ \\
3. Inconsistency & $50 \%$ & $20 \%$ & $30 \%$ \\
4. Feel inferiority & $80 \%$ & $10 \%$ & $70 \%$ \\
5. Inability to control her desires & $90 \%$ & $60 \%$ & $30 \%$ \\
6. Home situation & $50 \%$ & $10 \%$ & $40 \%$ \\
7. Being scared & $100 \%$ & $50 \%$ & $50 \%$ \\
8. Exam Anxiety & $50 \%$ & $10 \%$ & $40 \%$ \\
\hline
\end{tabular}

Note: Pre-post difference showed after the application of relaxation therapy that there was a great amount of reduction in psychological problems that were related to anxiety and depressive symptoms. 